\title{
One-Dimensional Scanning Approach to Shock Sensing
}

\author{
Roger Tokars and Grigory Adamovsky \\ NASA Glenn Research Center, Cleveland, Ohio, 44135 \\ and \\ Bertram Floyd \\ Sierra Lobo / NASA Glenn Research Center, Cleveland OH, 44135
}

\begin{abstract}
Measurement tools for high speed air flow are sought both in industry and academia. Particular interest is shown in air flows that exhibit aerodynamic shocks. Shocks are accompanied by sudden changes in density, pressure, and temperature. Optical detection and characterization of such shocks can be difficult because the medium is normally transparent air. A variety of techniques to analyze these flows are available, but they often require large windows and optical components as in the case of Schlieren measurements and/or large operating powers which precludes their use for in-flight monitoring and applications. The one-dimensional scanning approach in this work is a compact low power technique that can be used to non-intrusively detect shocks. The shock is detected by analyzing the optical pattern generated by a small diameter laser beam as it passes through the shock. The optical properties of a shock result in diffraction and spreading of the beam as well as interference fringes.

To investigate the feasibility of this technique a shock is simulated by a $426 \mu \mathrm{m}$ diameter optical fiber. Analysis of results revealed a direct correlation between the optical fiber or shock location and the beam's diffraction pattern. A plot of the width of the diffraction pattern vs. optical fiber location reveals that the width of the diffraction pattern was maximized when the laser beam is directed at the center of the optical fiber.

This work indicates that the one-dimensional scanning approach may be able to determine the location of an actual shock. Near and far field effects associated with a small diameter laser beam striking an optical fiber used as a simulated shock are investigated allowing a proper one-dimensional scanning beam technique.
\end{abstract}

\section{Introduction}

Numerous studies have been performed on shocks and their effects on performance of supersonic vehicles and their components ${ }^{1,2}$. Attempts have also been made to detect and monitor shocks in flight including normal shocks generated in a mixed compression supersonic inlet ${ }^{3-6}$.

As speed of vehicles increases approaching hypersonic regimes, formation of shocks and their interaction gain even more importance and the need to develop in-flight shock sensing and mitigation technology becomes even more acute ${ }^{7-10}$.

Various schemes developed to analyze high speed flows and evaluate performance of aircraft and spacecraft components have included such flow visualization techniques as shadowgraphs, Schlieren, and interferometry ${ }^{11-13}$.

Despite their attractiveness these tools usually involve powerful lasers and large optical components and therefore are not applicable to on-board applications. An alternative would be to use a scanning laser beam approach. Previously conducted experiments have demonstrated a significant change in the beam profile as a result of laser beam interaction with a shock ${ }^{14,15}$.

The changes in the laser beam profile upon interaction with the shock have led to the introduction of a scanning beam technique ${ }^{16}$. It has also been shown that similar changes in the laser beam profile can be generated by placing an optical fiber in the path of the beam ${ }^{17}$. In the present paper we have extended this 
approach and employed a one-dimensional scanning technique for sensing the relative position of an optical fiber and the incident laser beam.

\section{Beam Propagation through Interfaces}

Propagation of optical beams through various media is associated with such basic phenomena known from the electromagnetic field theory and optics as transmission, reflection, and refraction ${ }^{\mathbf{1 8}, 19}$. In addition, in the areas where an incident optical beam encounters boundaries or interfaces between media with different refractive indices a phenomenon called diffraction may occur. The geometrical theory of diffraction has been well described by Keller ${ }^{20}$ and extended to transparent smooth ${ }^{21}$ and straight edge ${ }^{22}$ objects. According to the theory, an incident beam represented by a plane wave and passing through or near such diffracting interface may be thought of in three parts. One of them continues its propagation in the medium it was originated in, the second one enters into the medium with a different refractive index and experiences reflection and refraction according to the Snell's Law ${ }^{23}$. The third part of the incident beam experiences diffraction. All three parts interfere with each other forming characteristic scattering patterns ${ }^{22}$

The underlying physics of the phenomenon still remains the same even when the incident plane wave is replaced by a Gaussian beam. The resultant patterns, however, are different. The differences have been shown mathematically for the case of Fraunhofer diffraction of a Gaussian wave field by a thin wire ${ }^{24}$ and dielectric cylinder ${ }^{25}$ as well as propagation of Gaussian beams through inhomogeneous media with shocklike profiles of refractive index ${ }^{26}$. The mathematical analysis has shown splitting of the incident Gaussian beam, redistribution of intensities between diffraction peaks, formation of multiple fringes and beam spreading.

Effects of abrupt interfaces on Gaussian beams predicted by the theory have also been demonstrated experimentally on aerodynamic shock waves ${ }^{14}$. Figure 1 shows an experimental setup to observe scattering of a Gaussian laser beam from a bow shock generated by a flow of air impinging in a bluff cylinder. Images of a Gaussian beam observed in both, the absence and presence of a shock are shown in Figures 2(a) and 2(b) respectively.

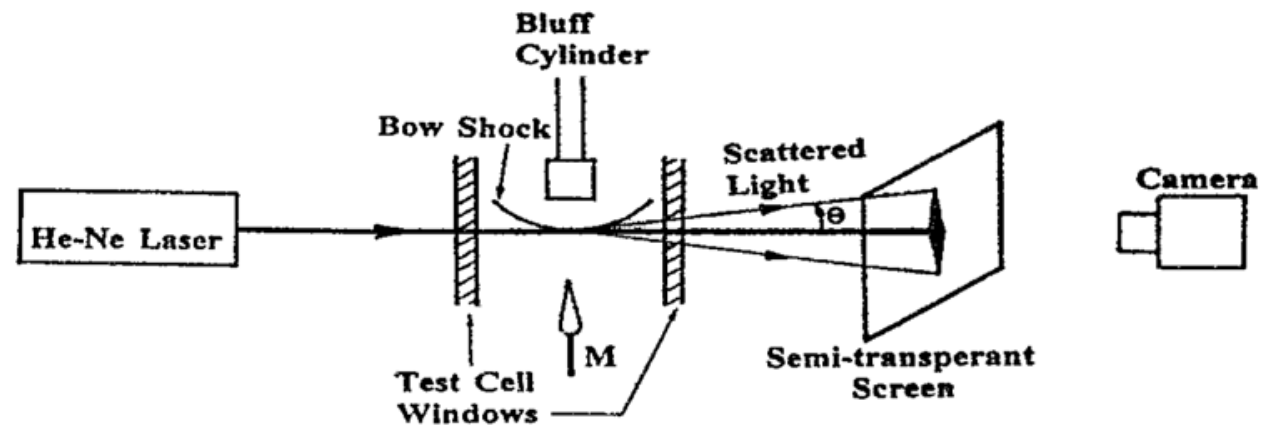

Figure 1. Aerodynamic test flow setup with laser (adopted from Ref. 14).
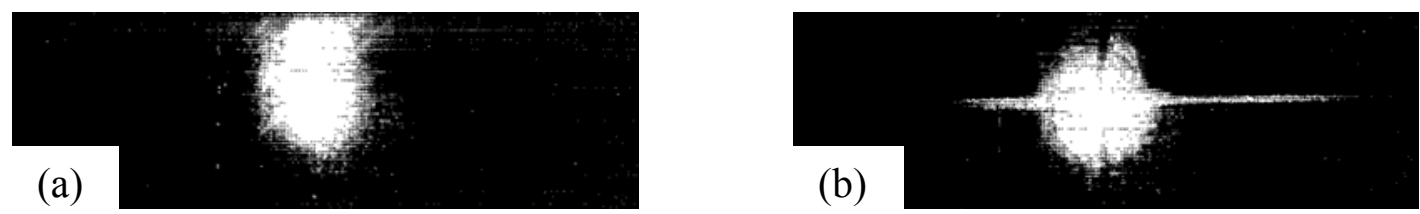

Figure 2. Images of a Gaussian beam diffracted by a bow shock as shown in Figure 1 in the absence, (a), and presence, (b), of a shock (adapted from Ref. 14).

The case of a laser beam interacting with a dielectric cylinder at grazing incidence is shown in Figure 3. In this demonstration, a 1-inch diameter dielectric cylinder is placed in the path of a laser beam and the 
pattern is observed in the plane located right behind the cylinder (Figure 3(b)) and at a distance of about $18 \mathrm{~mm}$ (Figure 3(a)).
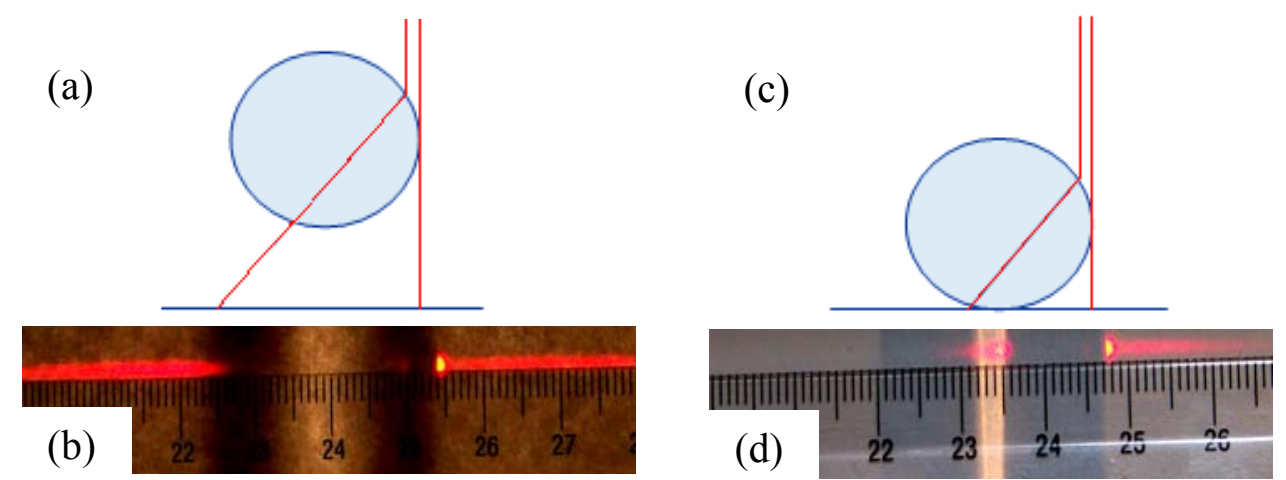

Figure 3. Scattering of a Gaussian beam striking a dielectric cylinder at grazing incidence observed at different locations of the observation screen: (a)-(b) Observation screen is at $18 \mathrm{~mm}$ behind the cylinder; (c )-(d) Observation screen is right behind the cylinder.

This demonstration shows a significant splitting of the incident beam to a degree such that the two beams are completely separated. Accompanying tails are also clearly seen. Moreover, a greater split between the resultant beams is observed at larger distances. In comparison with the pattern observed in Figure 2(b) this phenomenon of a large split between the resultant beams in Figure 3 can be explained by the significant difference in the refractive indices of the air and the dielectric cylinder. In the case of an aerodynamic shock, however, the refractive indices on both sides of the shock do not differ significantly from each other. Thus, a single interface as shown in Figure 3 does not generate a diffraction pattern similar to the one produced by a shock.

Another case of wave propagation through objects involves two interfaces. Examples of objects with double interfaces include a slit and a wire. Diffraction of plane waves and Gaussian beams from both a slit and a wire has been investigated intensively and the results, both analytical and experimental, can be found in the literature ${ }^{27-30}$. Figure 4 shows diffraction of a laser beam by an optical fiber obtained during our experiment.

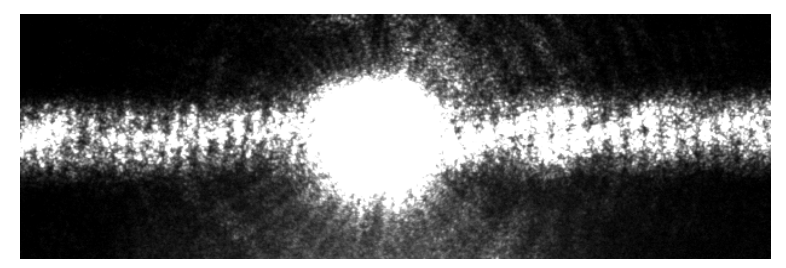

Figure 4. Diffraction of laser beam by an optical fiber.

Both cases of diffraction, from one and two interfaces, have common characteristic features. They both result in formation of a shadow and spreading of the incident beam with formation of tails.

\section{Scanning Beam Technique}

\section{III.A. Principle of Operation}

Shock detection using a scanning laser beam is based on scattering of the beam passing through aerodynamic shocks that have rapid changes in refractive index. Profiles of refractive index of aerodynamic shocks have been analyzed and in most cases approximated as an abrupt interface ${ }^{31,32}$. As was mentioned above, experimental results as well as computational analyses have described the significant changes in the beam profile that result from those interactions ${ }^{14,26}$. These changes manifest in the beam spreading and formation of tails and secondary fringes described above. Using these changes in the laser 
beam profile as an indicator of the presence of shocks, a shock detection technique based on a scanning of a small diameter laser beam has been devised. Reference 16 describes the design of the beam scanner which includes a mechanism to rotate the beam and to convert the rotation into translation. The changes in the beam structure are then detected and processed and the location of the aerodynamic shock can be determined based on the geometry of the installation.

This paper concentrates on the development of signal processing schemes to detect variations in the diffraction patterns generated by a laser beam passing through interfaces.

\section{III.B. Experimental Setup}

A schematic of the experimental setup used in this work is shown in Figures 5 and 6. It consists of a $0.5 \mathrm{~mW}$ Helium Neon (HeNe) CW laser, vertically positioned optical fiber, screen, and camera (See Figure 5). All major components are placed on a rail that permits change of the relative distance between them. The laser emits a $0.48 \mathrm{~mm}$ diameter beam with Gaussian profile at $0.6328 \mu \mathrm{m}$ wavelength. The optical fiber is a standard communication grade fiber with cladding diameter of $426 \mu \mathrm{m}$. It is placed on a translation stage with a transverse computer-controlled actuator. Such an arrangement permits changing the relative position of the fiber and the laser beam in a plane normal to the direction of the laser beam propagation. The diffraction pattern is projected onto a screen which is located $1250 \mathrm{~mm}$ away from the vertical fiber. A $105 \mathrm{~mm}$ focal length lens and 2x teleconverter (See Figure 6) are used to image the screen onto the $1024 \mathrm{x}$ $1024 \mathrm{CCD}$ imaging array with $13 \mu \mathrm{m} \times 13 \mu \mathrm{m}$ pixels. Images are captured in tagged image file (TIF) format with 8-bit resolution.

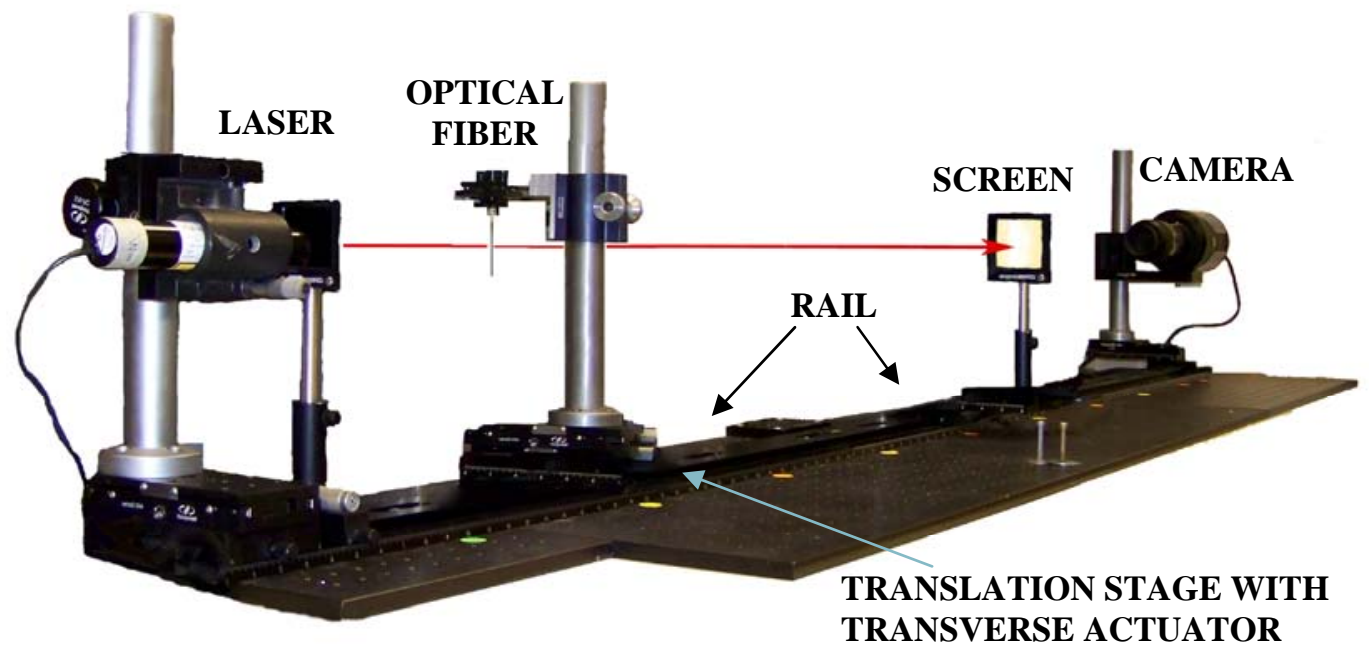

Figure 5. The experimental setup for the 1D scanning approach of an optical fiber.

Distances between major components are presented schematically in Figure 6. The schematic gives a projection of the setup on the horizontal plane. The optical fiber and the screen are positioned at approximately $640 \mathrm{~mm}$ and $1890 \mathrm{~mm}$ from the laser respectively.

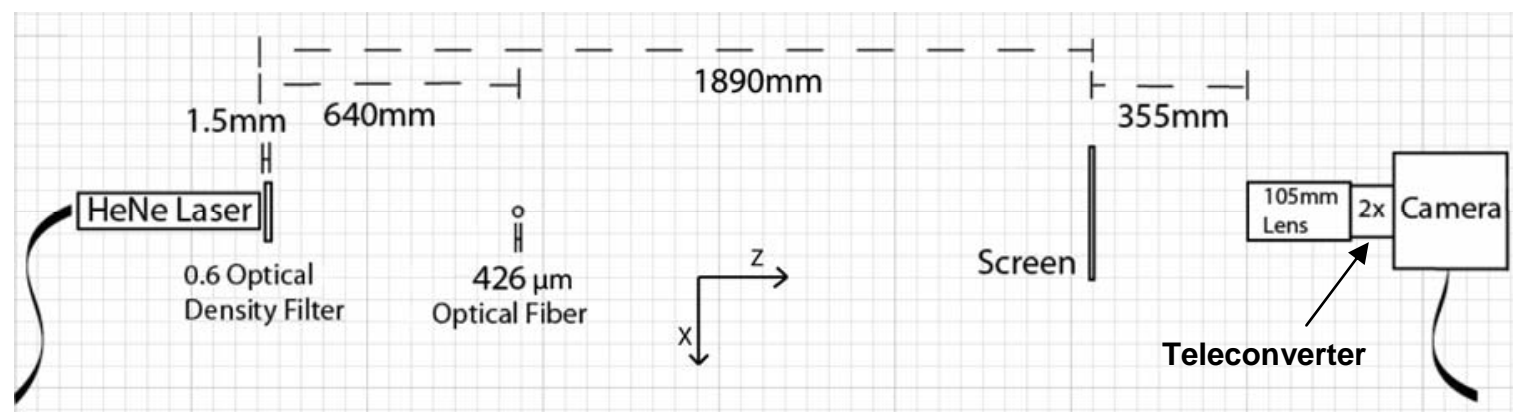

Figure 6. Drawing of the setup showing distances between major components. 
Several adjustments have been made to optimize the beam imaging with the CCD camera so that the entire beam and extended scattering effects are captured. The image of the beam is desired to be as large as possible to provide sufficient resolution for observing any changes in the diffraction pattern. The camera lens and teleconverter are adjusted to provide an imaged beam diameter that takes up a significant portion of the captured image (see Figure 7). Next, the intensity is reduced so that it does not saturate the camera sensors. This is accomplished by using a neutral density filter with optical density $\mathrm{OD}=0.6$, decreasing the aperture of the camera, and reducing the exposure time.

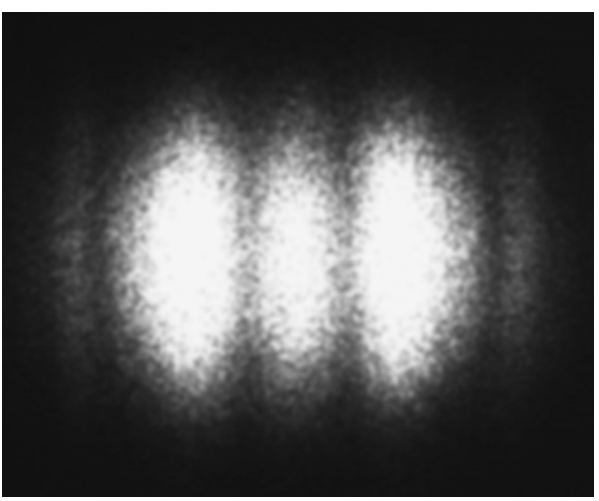

Figure 7. Laser images observed in setup of Figure 6 (with teleconverter) involving beam diffraction by an optical fiber at the center of the beam.

The laser beam can be approximated as an ideal Gaussian beam with parameters dependent on the distance from the laser ${ }^{33}$.

$$
\begin{gathered}
Z_{R}=\frac{\pi \omega_{o}^{2}}{\lambda} \\
2 \omega(z)=2 \omega_{0}\left[1+\left(\frac{Z}{Z_{R}}\right)^{2}\right]^{1 / 2}=2 \omega_{0}\left[1+\left(\frac{\lambda z}{\pi \omega_{o}^{2}}\right)^{2}\right]^{1 / 2},
\end{gathered}
$$

where $2 \omega_{o}$ is the diameter of the laser beam at the exit aperture,

$\lambda \quad$ is the wavelength of light,

$Z \quad$ is a variable distance from the laser exit aperture,

$Z_{R} \quad$ is the Rayleigh range which is defined as the distance over which the beam radius spreads by a factor of $\sqrt{2}$.

$2 \omega(z) \quad$ is the diameter of the laser beam at the distance $z$.

Using the equations above and properties of the laser, parameter values are calculated using the setup shown in Figure 6. The calculated Rayleigh range is approximately $286 \mathrm{~mm}$. Calculated from Eqs. 1 and 2, the diameter of the incident Gaussian beam at the location of the optical fiber and on the screen is approximately $1.18 \mathrm{~mm}$ and $3.21 \mathrm{~mm}$ respectively.

Adjustments to the camera magnification were selected to generate two different types of the imaged diffraction pattern. One type of the imaged pattern was produced with a teleconverter attached to the camera as shown in Figure 6. The teleconverter restricted the field of view. Such arrangement led to a narrow field of view and the resultant image was of a portion of the pattern near the center of the incident beam. The other type of imaged pattern was generated when the teleconverter was removed. That led to a wider portion of the pattern on the screen to become viewable by the camera. The wide field of view, also called a wide angle case, allowed the camera to capture the diffraction pattern not just at the center around the incident beam but also well beyond its boundaries. Figures 7 and 4 show images of a diffraction pattern 
obtained by the narrow and wide views respectively. The wide field of view captures a much larger area on screen than the narrow view which leads to a greater magnification of the area observed. In our experiment the area of observation was increased by a factor of 25 .

Inspection of a Gaussian beam diffracted by an optical fiber was done by replacing the screen in Figure 5 with a beam analyzer, a CCD based camera directly exposed to the image forming optical beam.

Movement of the fiber from the original location in Figure 6 to a position closer to the laser corresponding to $d_{\text {beam }} \sim \mathrm{d}_{\text {fiber }}$ results in a diffraction pattern with distinct dark and bright lobes moving father and father from the laser spot center. This is accompanied by decrease in the intensity of the diffraction pattern and an increase in the lobe separation. Movement of the fiber from the original location farther away from the laser, corresponding to the $\mathrm{d}_{\mathrm{beam}}>\mathrm{d}_{\text {fiber }}$, results in a smaller portion of the incident light passing through the fiber. The result is formation of an image of the fiber similar to observed by using a shadowgraph. Figure 8 demonstrates this phenomenon. It depicts intensity profiles of the laser beam diffracted by the optical fiber positioned at approximate distances of $0.25,0.75$, and $1.25 \mathrm{~m}$ from the laser. The beam analyzer is located at a fixed position of approximately $1.5 \mathrm{~m}$ from the laser.

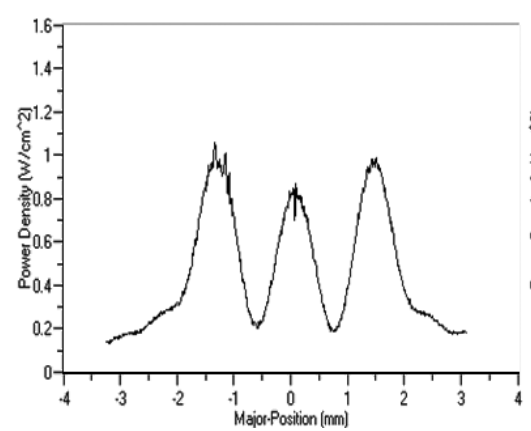

a)

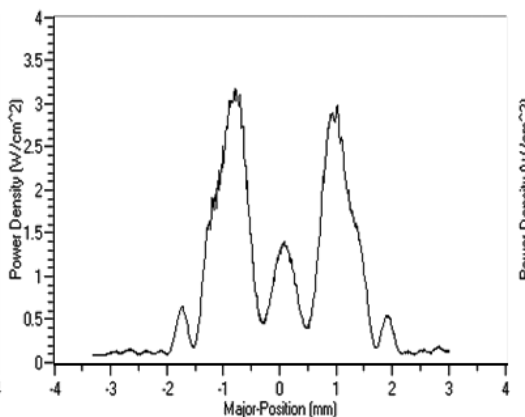

b)

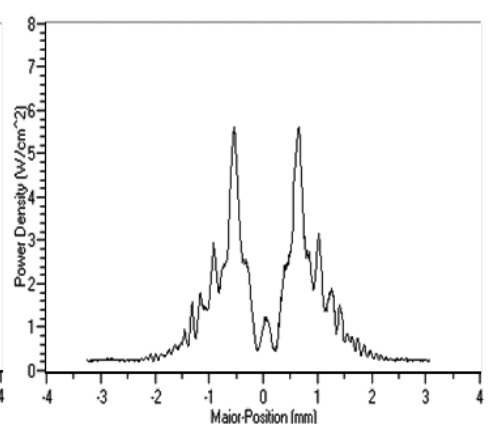

c)

Figure 8. Intensity profiles of diffraction patterns observed at a distance $1.5 \mathrm{~m}$. from the laser:

a) Fiber positioned at $0.25 \mathrm{~m}$ from the laser;

b) Fiber positioned at $0.75 \mathrm{~m}$ from the laser;

c) Fiber positioned at $1.25 \mathrm{~m}$ from the laser.

We also analyzed an intensity profile of a Gaussian beam with and without interaction with the fiber. The beam analyzer in this case was located in place of the screen in Figure 6. Also the neutral density filter was removed. Figure 9(a) shows the unobstructed laser beam profile. Figure 9(b) shows the profile of the laser beam with an optical fiber at the center of the laser beam path. The optical fiber location is indicated with the white line in Figure 9(b).
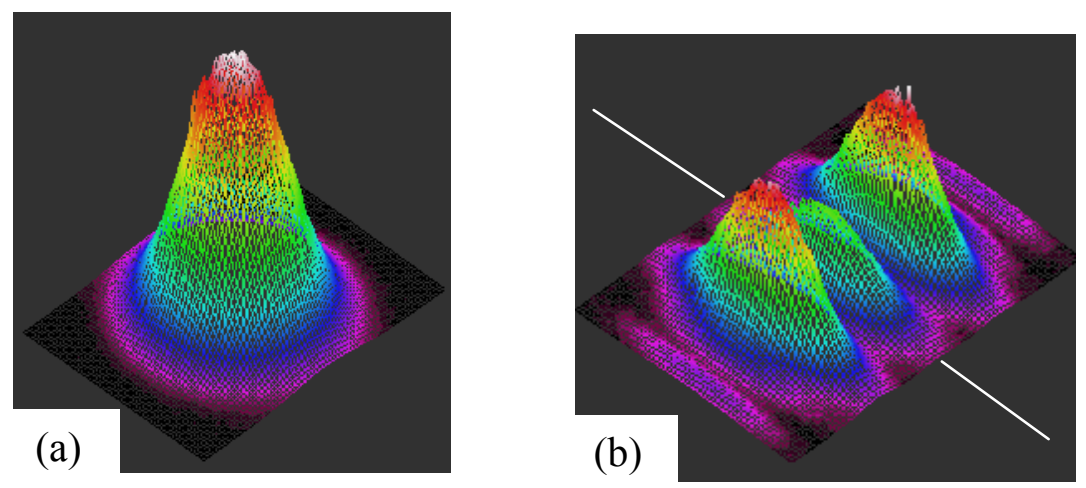

Figure 9. 3D Gaussian beam profile of (a) unobstructed laser and (b) laser with optical fiber in center. 
The Power Density measurements from Figure 9(a) and across the diffraction of Figure 9(b) are shown in Figure 10 below.

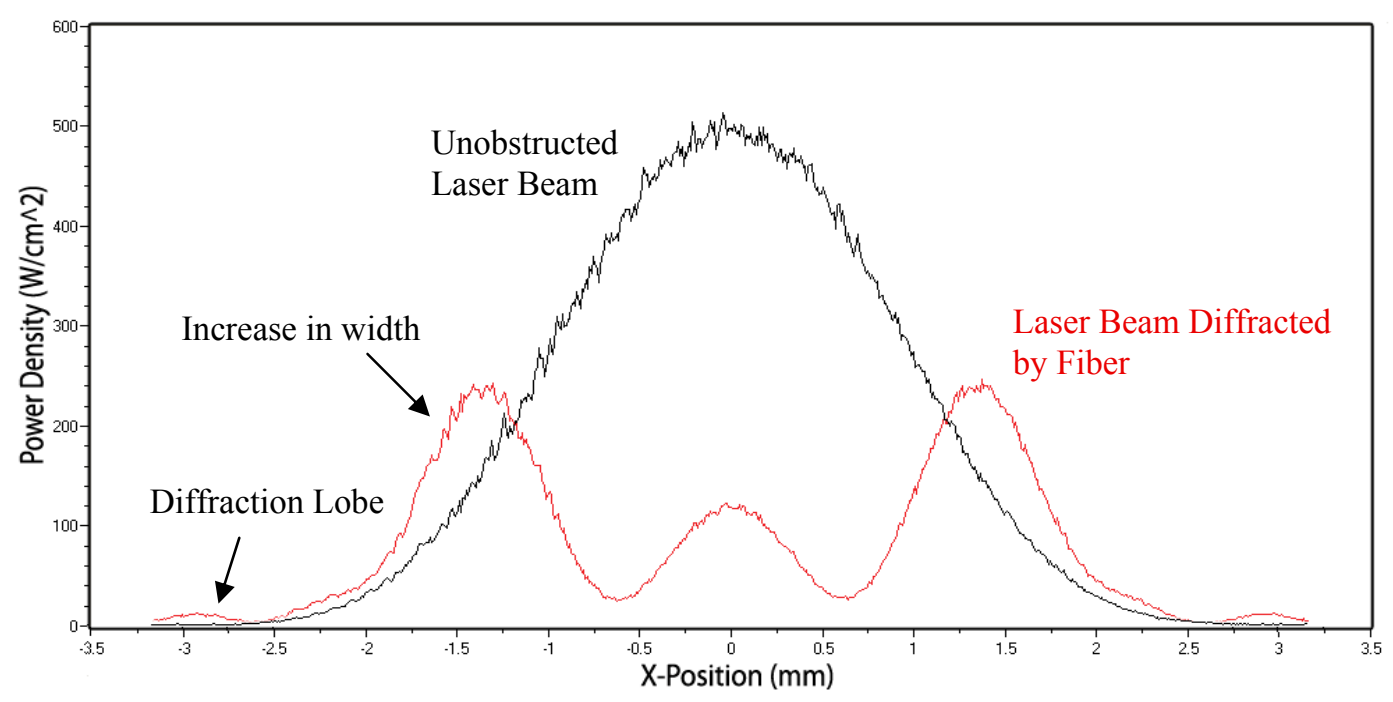

Figure 10. Gaussian beam profile comparison of the Power Density for a diffracted laser beam.

Figure 10 shows that the diffracted laser beam increases in width when compared to the unobstructed laser beam. Also the integration of areas under the curves shows an approximate $50 \%$ decrease in intensity in the diffracted pattern as compared to the intensity of the unobstructed beam. The losses can be attributed primarily to the wide angle scattering, and to back reflection and absorption. The redistribution of light intensity due to the wide angle scattering is shown in Figure 10 by the presence of the diffraction lobes. These lobes are visibly evident beyond the observed range in Figure 10 and have been previously shown in Figure 4.

For purposes of this paper the central portion of the observed laser beam pattern will be defined as the core beam. This is where the majority of the power of the laser beam is located. In this work, the core beam spread is investigated with the narrow view. Outside of the core beam, diffraction from the optical fiber reveals many secondary diffraction lobes of much lower intensity. In this work, the wide angle diffraction is investigated with the wide view.

\section{III.C. Image Processing}

The primary focus of this work is to develop image processing techniques to accurately detect and localize a shock using the diffraction signature described above.

Two image processing techniques have been developed, Consecutive Pixel Counting (CPC) and Summing Pixel Counting (SPC) techniques. These techniques are designed to detect the spreading of the laser as it passes through inhomogeneity such as an optical fiber or simulated shock. The CPC technique utilizes the narrow view, while the SPC technique utilizes the wide view.

\section{III.C.1. Consecutive Pixel Counting Technique}

CPC results in a quantitative value that estimates the width of the spread in terms of consecutive bright pixels. First, an intensity threshold value is selected between the dark minimum and bright maximum range of intensities of the camera. This threshold determines what intensity is bright enough to be considered part of the beam spread. The image is then scanned horizontally one row at a time. Using the intensity threshold value, the number of consecutive bright pixels is counted and the maximum number and location of the center is returned. This maximum number is a value which is compared to the value of a reference image where no diffraction takes place.

Figures 11(a) and 11(b) show initial CPC testing which contrasts a reference unobstructed beam to a beam passing through the center of the simulated shock. The maximum consecutive beam spread shown in 
Figures 11(a) and 11(b) with the red line are 525 and 657 pixels respectively. Because the images have a resolution of $9.7 \mu \mathrm{m}$ per pixel, these values correspond to beam spreads of $5.09 \mathrm{~mm}$ for the unobstructed beam and $6.37 \mathrm{~mm}$ for the obstructed beam. The unobstructed beam diameter is significantly different from the calculated Gaussian beam diameter of $3.21 \mathrm{~mm}$ from Eqs. 1 and 2. This difference is likely due to screen diffusion, camera saturation effects, and the use of an intensity threshold value that was selected more for measuring diffraction changes than the diameter.
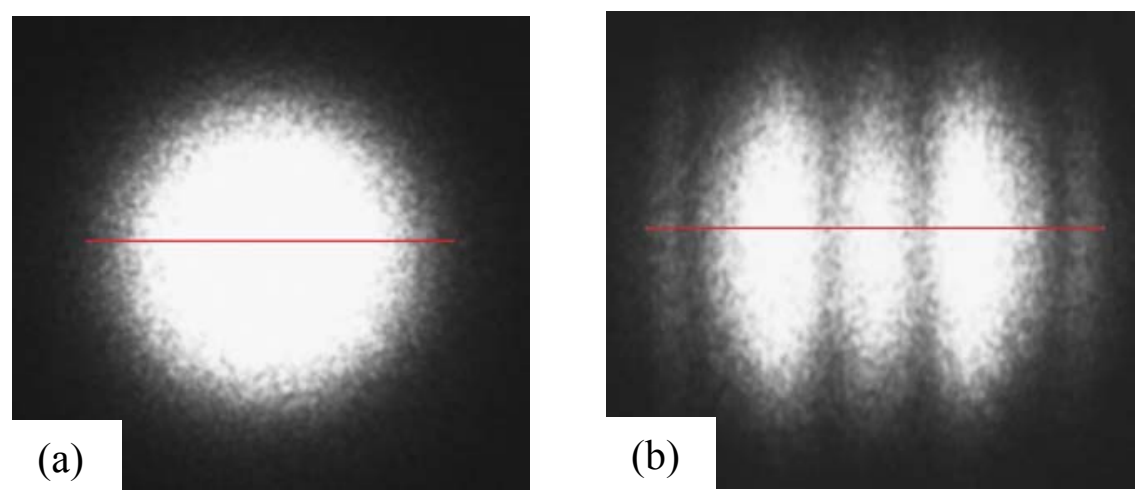

Figure 11. Laser images analyzed with the CPC technique involving an (a) unobstructed reference beam and (b) beam diffraction by an optical fiber or simulated shock at the center of the beam.

During image processing with the CPC technique, dark spots between the bright lobes in Figure 11(b) are problematic. One solution is to use a lower intensity threshold which can ignore the darker areas.

Another solution includes pre-processing the image with a 3 pixel Gaussian blur which helps combine the bright lobes together. The Gaussian blur is a weighted average blur. It adds low-frequency detail and can produce a hazy effect masking over the darker pixels with brighter pixels. This has the effect of making brighter pixels darker as well, but because much of the bright areas are already saturated, averaging with darker pixels along with the lower threshold value results in a successful detection of consecutive bright pixels.

During image acquisition, the blooming effect does cause some of the pixels directly above and below the beam to become bright as well. This bleeding effect occurs when the camera is exposed to a too high light intensity. Nonetheless, these saturation effects do not influence the results because the CPC and SPC techniques scan in the horizontal direction and blooming occurs in the vertical direction.

A more thorough test of the CPC technique involves a sequence of images captured to inspect how the simulated shock interacts with the laser beam. Using the setup in Figure 6, the simulated shock positioning begins outside the laser beam path. The simulated shock is then moved incrementally $15 \mu \mathrm{m}$

perpendicularly into the laser beam along the $X$ axis (see Figure 6). This movement increment was chosen because it is equivalent to the $15 \mu \mathrm{m}$ guaranteed on-axis accuracy of the actuator. An image is captured after each movement of the simulated shock. The testing continues until the simulated shock passes completely through the laser beam. The setup uses an optical density filter to reduce the intensity of the beam and an exposure time of $60 \mathrm{~ms}$ for the camera.

The results from processing the sequence of images as described above are shown in Figures 12 and 13. The maximum number of consecutive bright pixels determined by the $\mathrm{CPC}$ technique is plotted against the distance from the simulated shock. Distance $0 \mathrm{~mm}$, is determined visually as well as by the horizontal symmetry of the diffraction pattern as seen in Figure 11(b). 


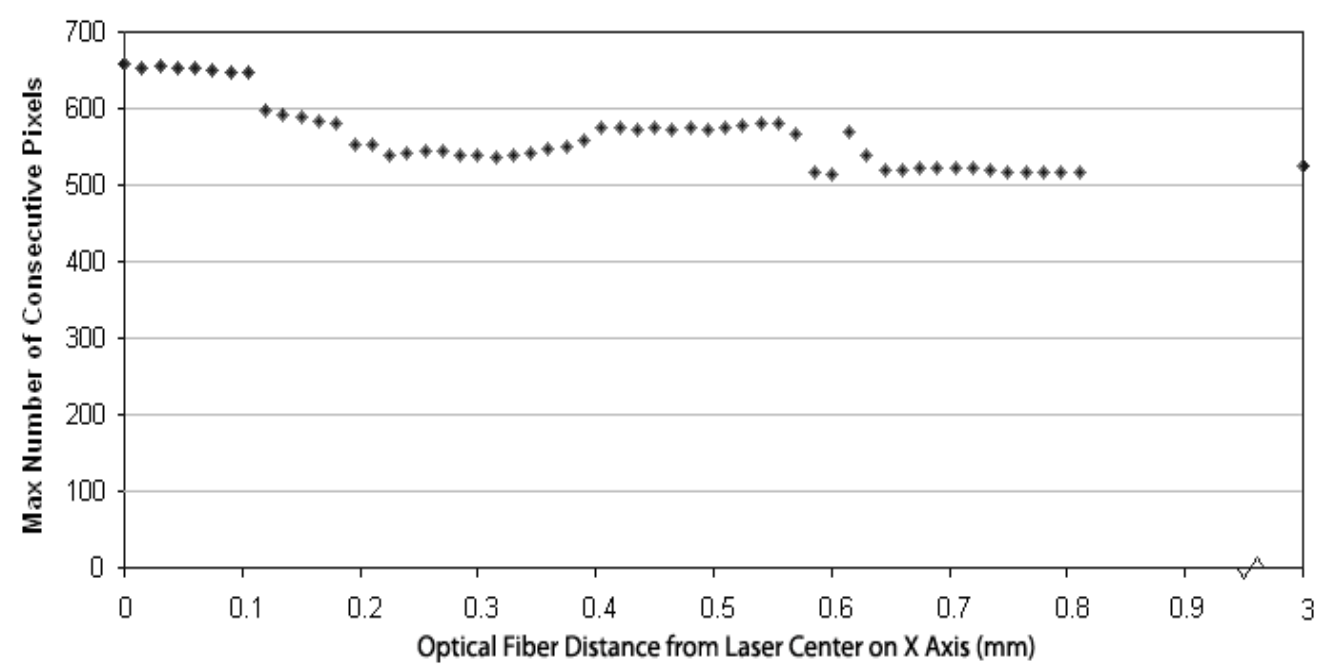

Figure 12. Dependence of the maximum number of consecutive pixels in the spread of the laser beam pattern in regards to the relative position of the optical fiber or simulated shock and beam along the $\mathrm{X}$ axis.

Figure 12 only shows data points up to the center of the laser beam path because the results are symmetrical around the $0 \mathrm{~mm}$ point. As the simulated shock displacement is increased beyond $0.8 \mathrm{~mm}$, the approximate spread in the laser beam pattern remains at 525 pixels. This is as expected because the simulated shock will no longer affect the beam when the simulated shock is outside the beam's path.

The images shown in Figure 13 illustrate the change in the diffraction pattern as the simulated shock enters the laser beam from the left side of the image. Figure 13(a) is the unobstructed reference beam and Figure 13(f) is the fully obstructed beam, which are also Figures 11(a) and 11(b) respectively. The red line indicates the location of the maximum consecutive bright pixels with the intensity threshold sensitivity set at 50. The text in the image shows the approximate location in $\mathrm{mm}$ of the simulated shock from the center of the laser beam as well as the length of the red line in pixels.
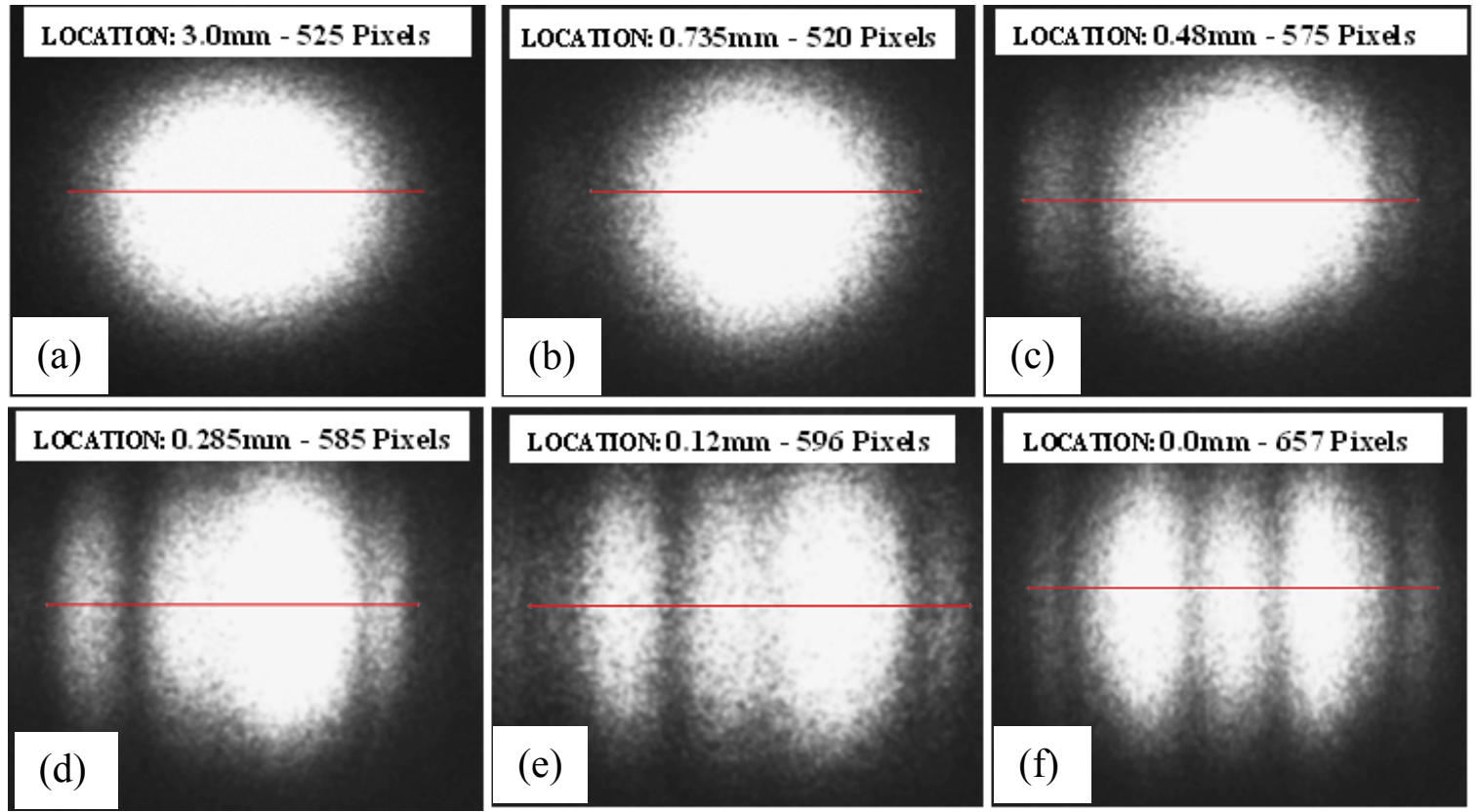

Figure 13. Gradual change in the laser beam pattern as the optical fiber or simulated shock is moved into the center of the beam from the left side of the image along the $X$ axis. 
The CPC technique's shortcomings are revealed in the results. Specifically the influence of disconnected bright lobes results in inconsistent consecutive bright pixels. The narrow field of view visualizes the detail of beam diffraction specifically near the center. This allows one to see where the dark spots disrupt the CPC technique.

\section{III.C.2. Summing Pixel Counter Technique}

The SPC technique emphasizes using all diffraction spreading such as that visualized through the wide camera view.

To mitigate the deficiencies in the CPC technique, the Summing Pixel Counter (SPC) technique has been devised. Much like the CPC technique, the SPC technique counts bright pixels that have an intensity value greater than or equal to a chosen intensity threshold, but where the SPC technique differs is that the bright pixels do not have to be consecutive. Instead, all the bright pixels are counted for each row and the maximum number and location is returned.

Figure 14 shows initial SPC testing of a beam passing through the center of the simulated shock. The maximum summed beam spread for a given intensity threshold is shown in red. The currently chosen threshold focuses on detection of the bright diffraction lobes in the central area of the image, whereas the dimmer lobes are mostly ignored. Reducing the threshold value would increase the sensitivity of the SPC technique to detect a larger portion of the diffracted light. As seen in Figure 14, intermittent dark areas have no effect on the bright pixel counting of the SPC technique, which is a major improvement over the CPC technique.

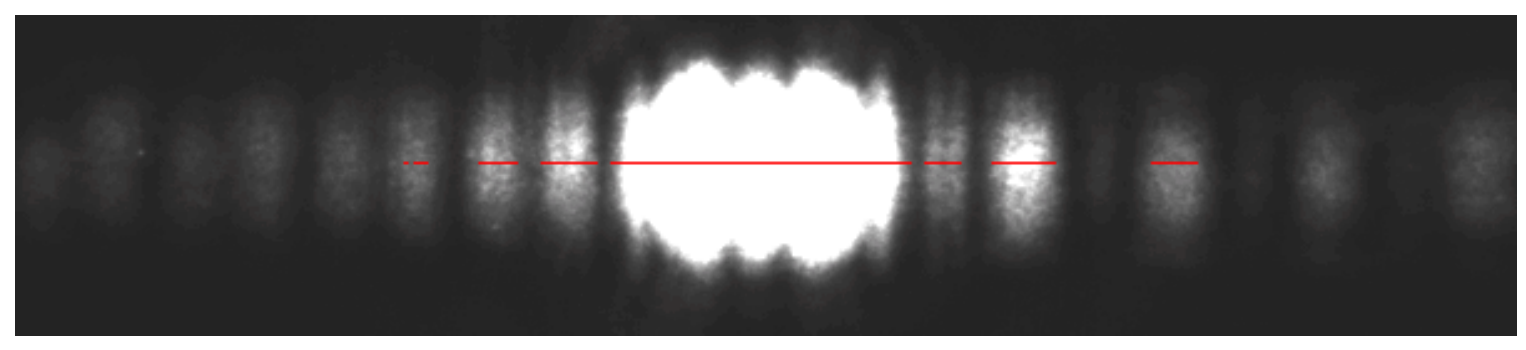

Figure 14. Wide view of beam diffraction by an optical fiber or simulated shock at the center of the beam analyzed with the SPC technique.

The testing for the SPC technique used the Figure 6 setup except for a few minor improvements. Just as in the previous testing of the CPC technique, the simulated shock is moved through the laser beam perpendicularly along the $\mathrm{X}$ axis, except that the incremental movement is increased to $50 \mu \mathrm{m}$ for the SPC testing. This is done to reduce the time required for testing, while still yielding acceptable results. The teleconverter is removed from the setup so that a larger field of view is exposed to the camera. Due to lab constraints, the camera and screen are moved $360 \mathrm{~mm}$ closer to the laser source. The resulting captured images have a resolution of $47.6 \mu \mathrm{m}$ per pixel. To increase the intensity of the diffraction pattern, the laser beam power is increased by removing the neutral density filter. This results in saturation of the image at the center. In order to create an image despite of saturation we employed the accumulation technique described in Ref. 34.

The results from processing a sequence of images with the simulated shock passing through the laser beam using the SPC technique are shown in Figure 15. The maximum number of bright pixels is calculated by the SPC technique. The distance is the location of the simulated shock in $\mathrm{mm}$ with respect to where the center of the beam passes through the center of the simulated shock. Distance $0 \mathrm{~mm}$ is determined visually as well as by the horizontal symmetry of the diffraction pattern as seen in Figure 14. While Figure 14 uses an intensity threshold of only 48, seven different intensity thresholds are plotted to demonstrate the sensitivity of the SPC technique in Figure 15. 


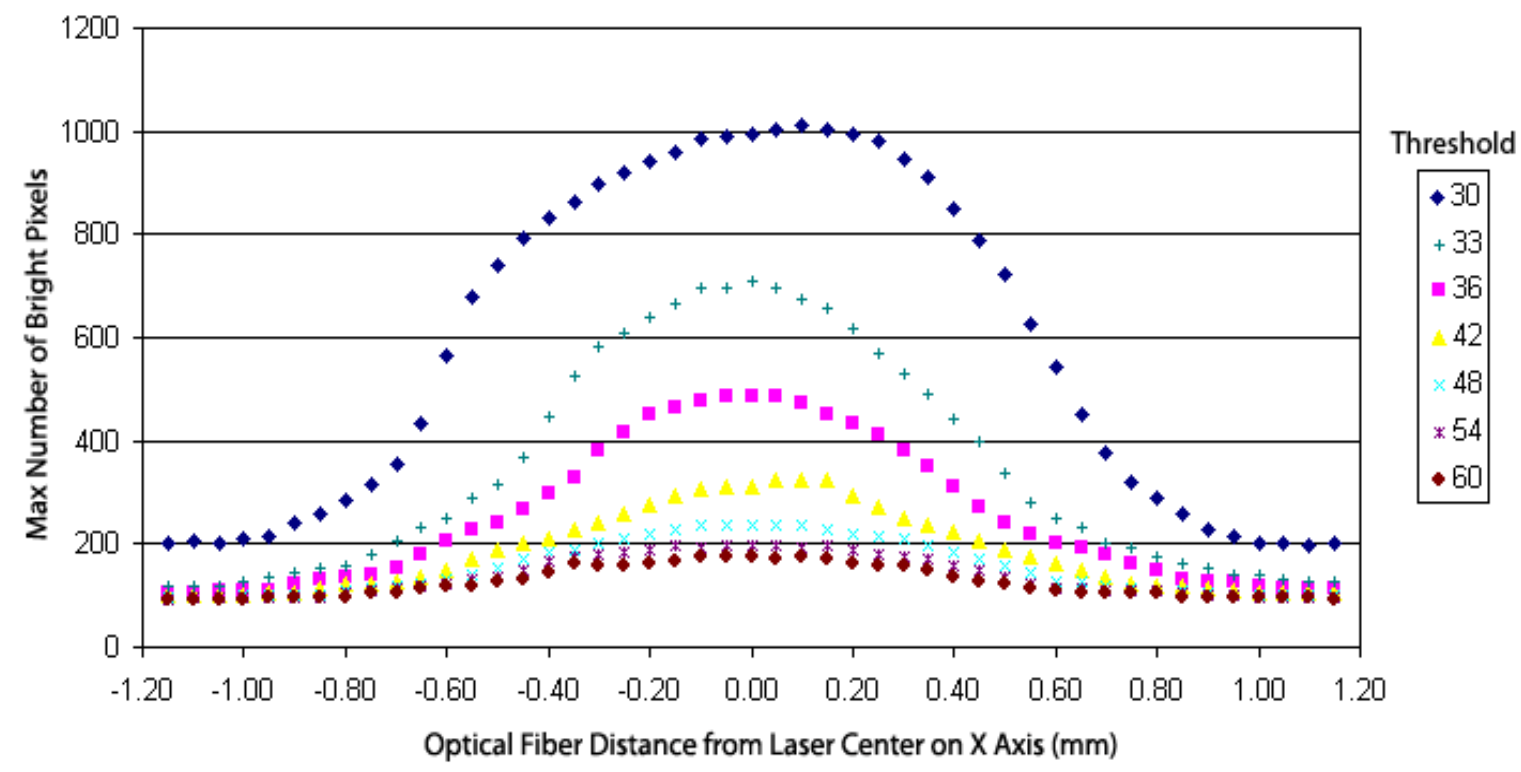

Figure 15. Dependence of the maximum number of pixels in the spread of the laser beam pattern on the relative position of the optical fiber or simulated shock and beam along the $X$ axis.

Figure 15 shows results of scans with various intensity thresholds ranging from 30 to 60 . Testing with this setup with thresholds below 30 began to show erroneous results because a lower threshold fell into the noise range for this setup, thus counting pixels as bright that would normally be considered dark.

Thresholds above 60 began to result in erroneous results because this threshold is beyond the intensity range of the diffracted light, thus ignoring pixels normally considered bright. The maximum number of bright pixels levels off beyond the simulated shock location range shown in the graph above because the simulated shock no longer affects the beam when the simulated shock is outside the beam's path.

The results from Figure 15 can be used to find the best possible intensity threshold by taking the maximum value and dividing by the minimum value for each threshold. In other words, this is the ratio of the diffracted beam over the reference beam. Figure 16 below shows the results of such calculations.

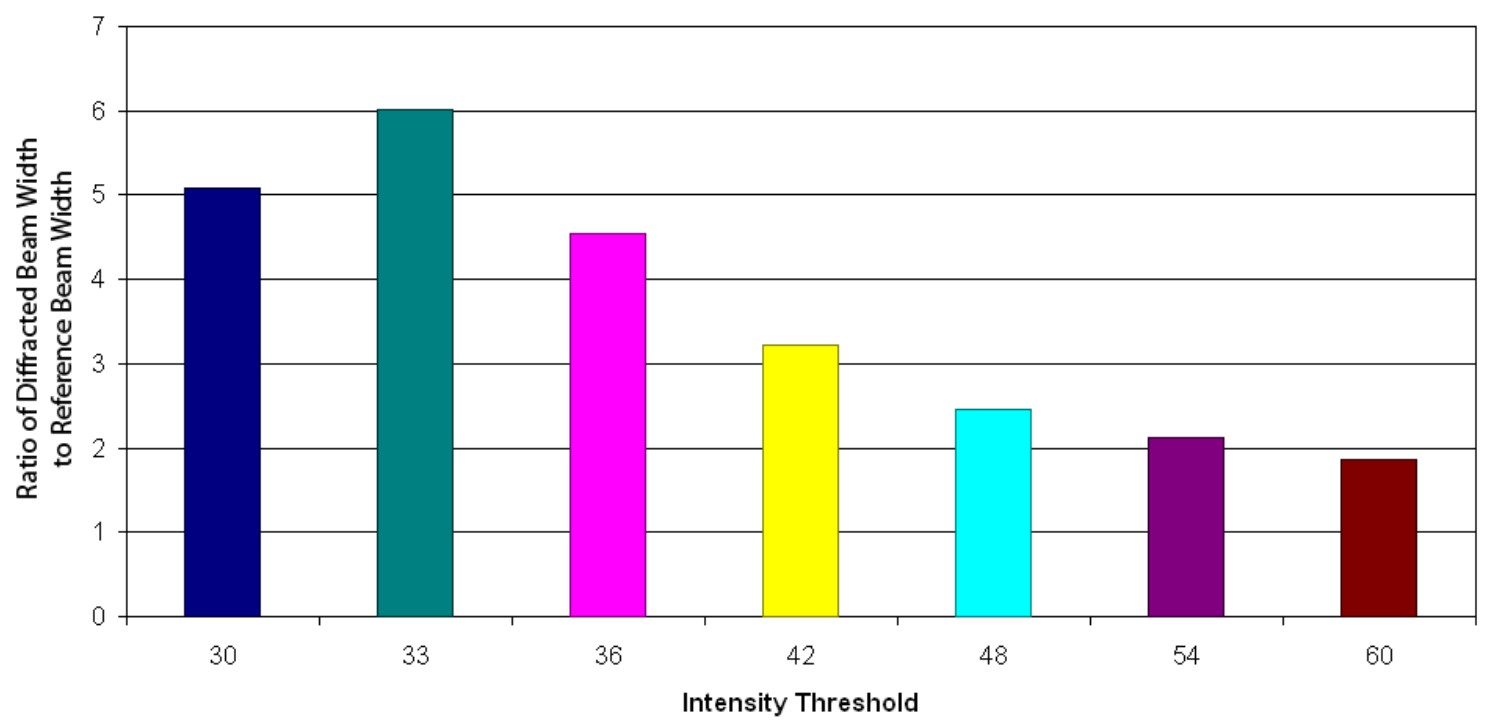

Figure 16. Different intensity ratios result in different calculated ratios of the diffracted beam width to the reference beam width. 
Figure 16 shows a peak ratio at an intensity threshold of 33 . This intensity threshold is the optimum choice in this experiment for determining the positioning of the simulated shock when comparing the diffracted beam to the reference beam.

\section{III.C.3. Near and Far Field Comparison of the Two Techniques}

A comparison of near and far field effects may be observed by moving the simulated shock different distances from the laser along the $\mathrm{Z}$ axis while still remaining in the center of the laser beam. The optical fiber or simulated shock was positioned at $17 \mathrm{~cm}$ away from the laser, then is moved to $20 \mathrm{~cm}$, then was incremented by $5 \mathrm{~cm}$ until it reaches the final position of $145 \mathrm{~cm}$. The $17 \mathrm{~cm}$ limits $145 \mathrm{~cm}$ increments are the two distance extremes that are possible without making contact with the other setup equipment. At each increment, the resulting diffraction pattern was captured and analyzed with the CPC and SPC techniques with an intensity threshold of 100. The results of these tests are shown in Figure 17.

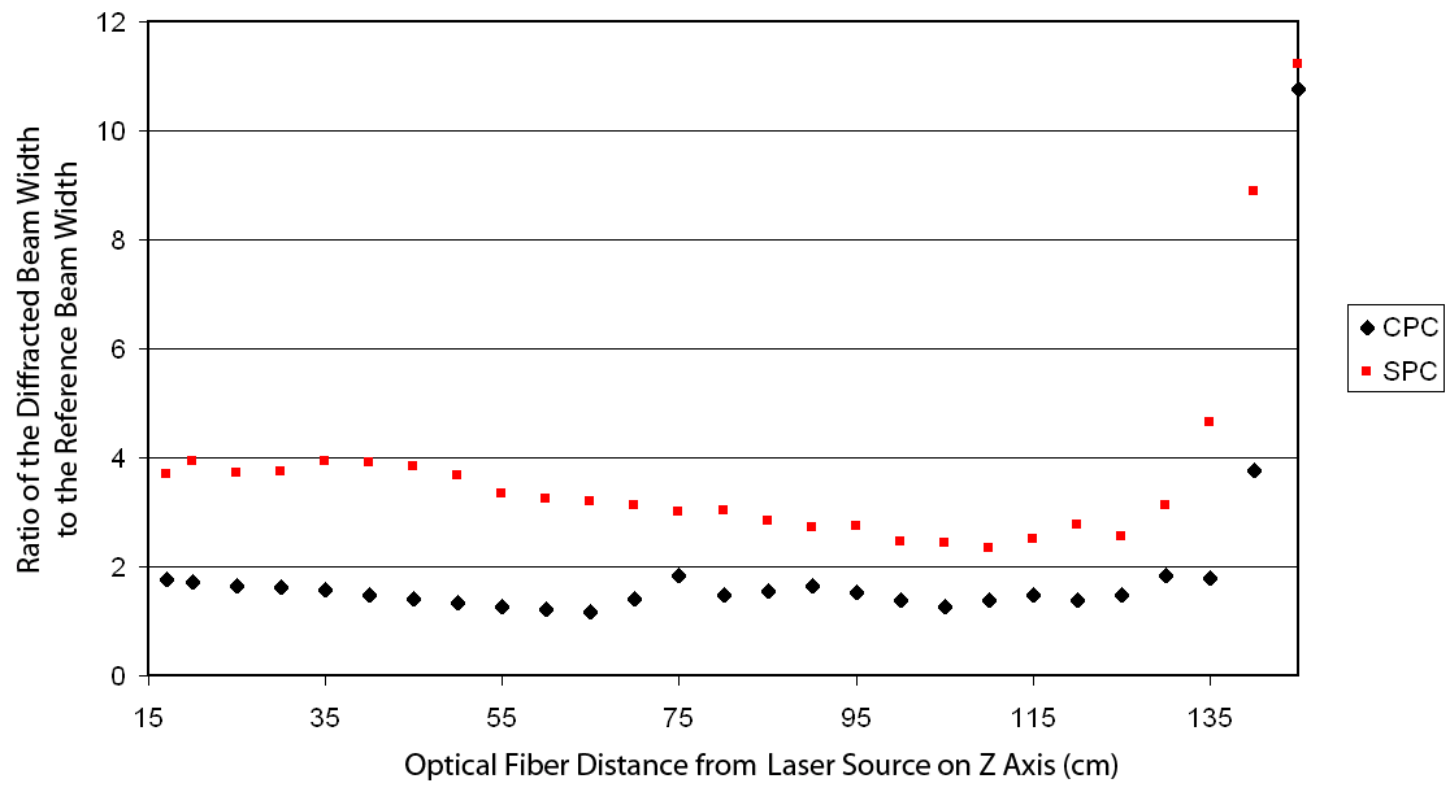

Figure 17. Ratio demonstrating the increase in the maximum number of pixels in the spread of the laser beam pattern in regards to the optical fiber or simulated shock location from the laser source along the $\mathrm{Z}$ axis.

The near and far field effects are inspected to ensure the reliability of the pencil beam shock sensing technique even when there are concerns of Rayleigh range influence. The Rayleigh range is the short distance after light exits the laser where the light is best modeled as a planar wave. After passing the Rayleigh range, the light is then best modeled as a spherical wave. The Rayleigh range of our setup is approximately $28.6 \mathrm{~cm}$. The results in Figure 17 across the entire range show a ratio greater than 1, which implies that the CPC and SPC techniques can effectively localize the simulated shock based on the diffraction of the laser beam regardless of the distance of the simulated shock from the laser. It is also obviously apparent that the SPC technique has a ratio that is greater than the CPC technique across the entire range of distance increments, thus implying that the SPC technique is an improvement to the CPC technique. A drastic increase in the calculated ratio from both the CPC and SPC techniques in the last four points is most likely caused by the simulated shock approaching the screen. While such an effect might seem inconsistent with the rest of the results, the ratio being greater than 1 still implies that the $\mathrm{CPC}$ and SPC techniques can be used to localize the simulated shock.

The diffraction pattern morphology differs with respect to the distance the optical fiber or simulated shock is to the laser, as seen in Figure 18. The diffraction pattern when the optical fiber is close to the laser has large distinct bright lobes, each with a larger width than those in the pattern where the optical fiber is farther away from the laser. Moving the optical fiber away from the laser results in the individual lobes moving towards the center of the laser beam, as if the diffraction pattern is squeezing itself together. The 
end result at $145 \mathrm{~cm}$, which is the furthest from the laser one could go in the setup used, is a blurred diffraction pattern, similar to Figure 18(c), without the existence of separate distinguishable lobes as seen in Figures 18(a) and 18(b).
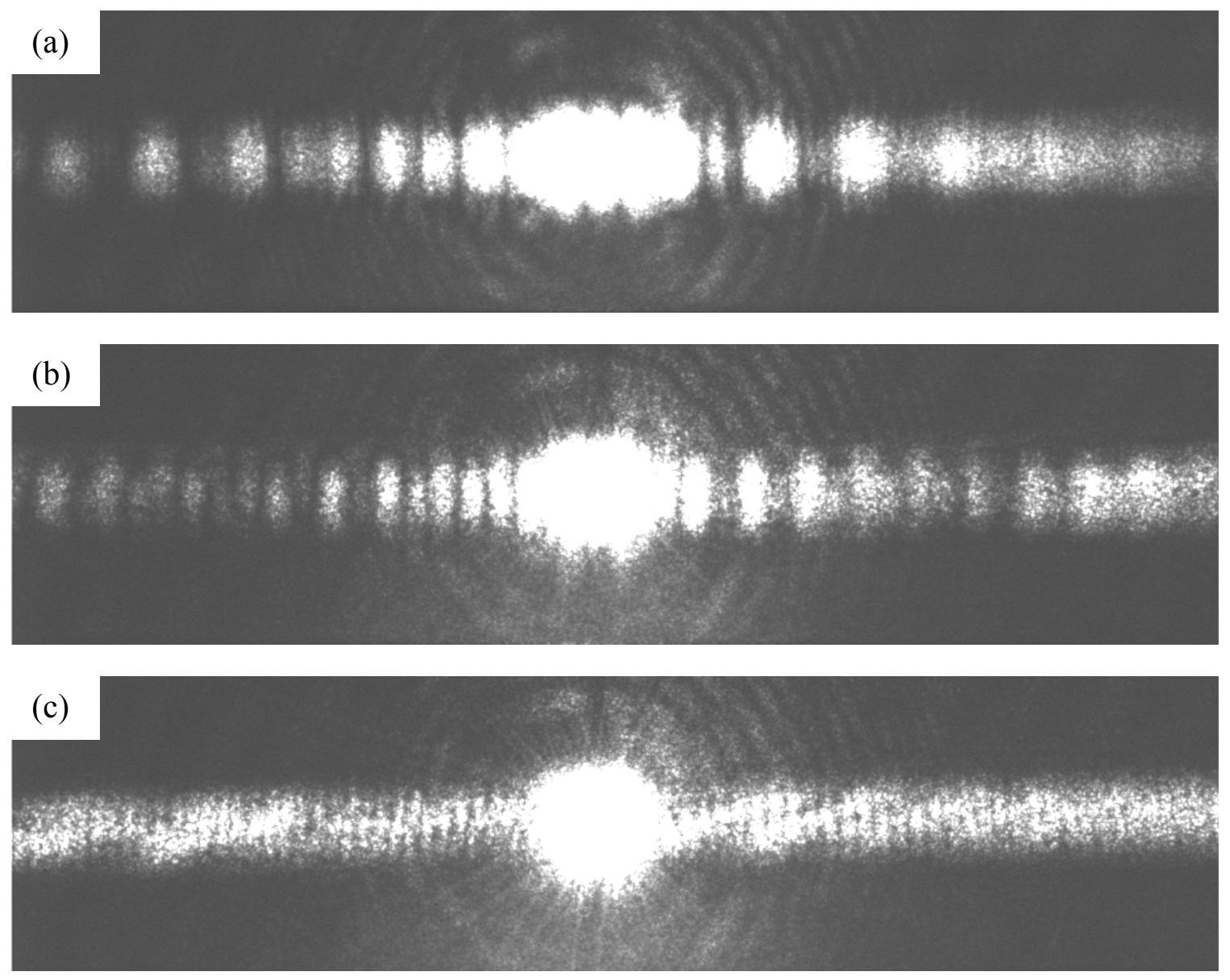

Figure 18. Diffraction pattern associated with the optical fiber or simulated shock at the center and approximate distance away from the laser source of (a) $30 \mathrm{~cm},(b) 80 \mathrm{~cm}$, and (c) $130 \mathrm{~cm}$ along the $Z$ axis.

\section{Discussion}

Visualization of the diffraction pattern on a screen can be described as the circular shape of the laser beam being distorted into several oval shaped lobes with different sizes and intensities. These lobes shift and recombine with each other as the optical fiber or simulated shock is moved across the laser beam (see Figure 13). The shifting and recombining of the lobes in the narrow view inspected in the CPC technique result in the laser beam spread width not being a monotonic function of the simulated shock movement. Specifically within the simulated shock displacement range of approximately $0.3 \mathrm{~mm}$ to $0.6 \mathrm{~mm}$ in Figure 12 , the laser beam spread width increases as the simulated shock is moved through this region. The reason for this is because of the shift in two smaller lobes relative to the bigger lobe in the center. One small lobe combines with the larger lobe decreasing the laser beam spread width disproportionately less than the other small lobe moving away and increasing the beam spread width. Figure 19 helps visualize this lobe interaction as the simulated shock is moved away from the laser beam center (W corresponds to the estimated beam spread width). Notice that in Figure 19(c), the small lobe is completely inside the larger lobe, thus it is not having any affect on the beam spread width $\mathrm{W}_{3}$. 

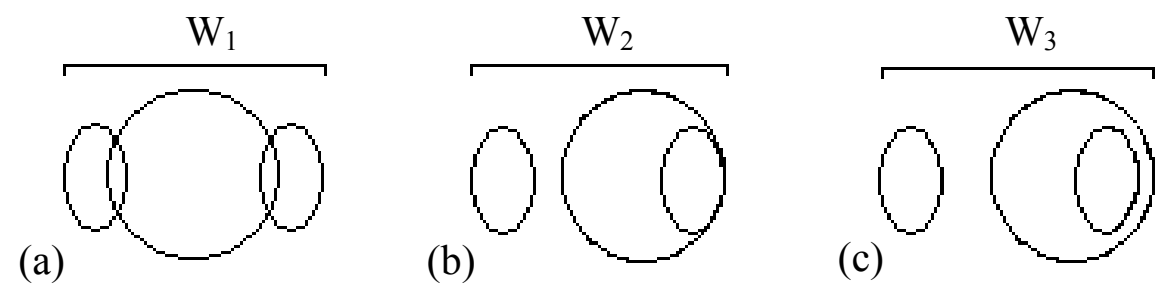

Figure 19. Lobe Interaction (Not to scale)

The diffraction lobe movement often results in dark areas in the images which interfere with the CPC technique. As is seen in Figure 12, within the simulated shock displacement range of $0.585 \mathrm{~mm}$ to $0.63 \mathrm{~mm}$ there is a discontinuity in the results. It's possible that the displacement points $0.585 \mathrm{~mm}$ and $0.6 \mathrm{~mm}$ have a dark spot interference causing the consecutive pixel counter to stop short. It's also possible that displacement points $0.615 \mathrm{~mm}$ and $0.63 \mathrm{~mm}$ have a bright speckle that connected two lobes for a longer than normal sequence of consecutive bright pixels. Either way, the sometimes random light speckles are an unpredictable variable that have a great affect on the CPC technique.

The SPC technique on a larger viewing screen is superior to the CPC technique in distinguishing when the laser beam goes through the center of the simulated shock. The SPC technique results, as seen in Figure 17, are consistently better than the CPC technique. Even better results for the SPC technique can be obtained if necessary by capturing the diffraction pattern that seemed to extend beyond the camera field of view. Figure 15 and 16 also show the benefits of tweaking the threshold sensitivity value on the results. Care needs to be taken to ensure that the threshold ignores noise of the system, such as ambient light sources, yet is sensitive enough to pick up the dim diffraction patterns.

Patterns seen in Figure 18 could also be used to evaluate the diffraction pattern around the core beam to calculate the distance the simulated shock is from the laser source assuming the distance the screen is from the laser source is fixed. Further investigation of the diffraction pattern is suggested to obtain a better positioning estimation of the simulated shock on the $\mathrm{Z}$ axis.

\section{Conclusions and Future Work}

Preliminary work on the one-dimensional scanning approach to shock sensing has been shown to have the capability to localize a simulated shock. Two techniques are demonstrated and evaluated to detect the location of the simulated shock using diffraction patterns. The technique based on a wide angle diffraction pattern has shown noticeable improvement in sensitivity. The effects of parameters such as distances between the main components of the setup, diameter of the laser beam, and profile of the simulated shock have been investigated. Automated image processing succeeded in quantifying the location of the simulated shock in an efficient manner. The steps taken to tackle various problems are explained. These preliminary results demonstrate the viability of one-dimensional scanning in supersonic and hypersonic wind tunnels to detect real aerodynamic shocks. Also, it has a potential to identify such shock properties as thickness, strength as well as location of the shock in the direction transverse to the flow. Moreover because of a small weight and size of components involved it has a potential of being used in airborne applications.

\section{Acknowledgement}

The work was supported by the Hypersonic Project under the NASA's Fundamental Aeronautics Program. The authors would also like to acknowledge Robert C. Anderson for his valuable advices on image processing and the help of James W. Williams in setting up the experimental optical systems. 


\section{References}

${ }^{1}$ Wilcox, F. A. and Perchonok, E., "Aerodynamic Control of Hypersonic Inlets for Optimum

Performance," NACA Research Memorandum, NACA RM E55L1, 1956.

${ }^{2}$ Cattafesta, L. N., III and Settles, G. S., "Experiments on Shock/Vortex Interaction", AIAA-92-0315

${ }^{3}$ Hewett, M. D., "High-Speed Civil Transport Issues and Technology Program," NASA CR-186020, 1992.

${ }^{4}$ Ray, J. K, Carlin, C. M., and Lambregts, "High-Speed Civil Transport Flight- Propulsion-Control

Technological Issues,” NASA CR-186015, 1992.

${ }^{5}$ Saiben, M, Donovan, J, F., and Morris, M. J., "Flight Prototype Normal Shock Sensor," NASA CR-18520, 1990.

${ }^{6}$ Iverson, D. G., Jr., and Daiber, T. D., "Flight Prototype Normal Shock Position Sensor", NASA CR185204, 1989.

${ }^{7}$ Bertin, J. J. and Cummings, R., M., "Fifty years of hypersonics: where we've been, where we're going", Progress in Aerospace Science 39, pp. 511-536 (2003).

${ }^{8}$ Wright, M. J., Sinha, K., Olejniczak, J., and Candler, G. V., "Numerical and Experimental Investigation of Double-Cone Shock Interactions", AIAA Journal, Vol. 38, No. 12, pp. 2268-2276 (2000).

${ }^{9}$ Adelgren, R. G., Yan, H, Elliott, G. S., Knight, D. D., Beutner, T. J., Zheltovodov, A. A., Ivanov, M., and Khotyanovsky, D., "Localized Flow Control by Laser Energy Deposition Applied to Edney IV Shock Impingement and Intersecting Shocks", Paper \# AIAA 2003-31.

${ }^{10}$ Albertson, C. W. and Venkan, V. S., "Shock Interaction Control for Scramjet Cowl Leading Edges," AIAA 2005-3289.

${ }^{11}$ Shapiro, A. H., The Dynamics and Thermodynamics of Compressible Fluid Flow, Volume I, The Ronald Press Company, New York, 1953, Chaps. 3.7.

${ }^{12}$ Merzkirch, W., Flow Visualization, $2^{\text {nd }}$ ed., Academic Press, New York, 1987.

${ }^{13}$ Verna, S. B. and Koppenwallner, G. "Detection of Shock Motion Using Laser Schlieren System in a Hypersonic SWBLI Flowfiield", AIAA 2001-1756.

${ }^{14}$ Panda, J. and Adamovsky, G., "Laser Light Scattering by Shock Waves," American Institute of Phys., Vol. 7, No. 9, September 1995, pp. 2271-2279.

${ }^{15}$ Adamovsky, G. and Johnson, D. K., "Optical Techniques for Shock Visualization and Detection," in Optical Techniques in Fluid, Thermal, and Combustion Flows, edited by S. S. Cha and J. D. Trolinger, SPIE Proceedings, Vol. 2546, 1995, pp. 348-357.

${ }^{16}$ Adamovsky, G., "Scanning Mode Shock Position Sensor Invented and Demonstrated", in Research \& Technology 1998, NASA/TM 1999-208815, 1999, p. 58.

${ }^{17}$ Adamovsky, G., Tokars, R., Varga, D., and Floyd, B. , "Wave Propagation through Inhomogeneities with Applications to Novel Sensing Techniques", Paper \# AIAA-2008-255-783.

${ }^{18}$ Balanis, C. A., Advanced Engineering Electromagnetics, John Wiley \& Sons, New York, 1989, Chaps. 4 and 5 .

${ }^{19}$ Gaskill, J. D., Linear Systems, Fourier Transforms, and Optics, John Willey \& Sons, New York, 1978, Chap. 10.

${ }^{20}$ Keller, J. B., "Geometrical Theory of Diffraction“, J. Opt. Soc. Am. Vol. 52, No. 2, pp. 116-130 (1962).

${ }^{21}$ Chen, Y. M., "Diffraction by a Smooth Transparent Object", J. Math. Physics 5, No. 6, pp. 820-832 (1964).

${ }^{22}$ Ohtsuka, Y. and Cheah, Y. M., "Fresnel diffraction by a semitransparent straight edge object with acoustically coherence-controllable illumination", Appl. Opt. Vol. 23, No. 2, pp. 300-306 (1984).

${ }^{23}$ Nussenzveig, H. M., "High-Frequency Scattering by a Transparent Sphere. I. Direct Reflection and Transmission”, J. Math. Phys. Vol. 10, No.1, pp. 82-120 (1969).

${ }^{24}$ Ganci, S., "Fraunhofer diffraction by a thin wire and Babinet's principle," Am. J. Phys., Vol. 73, No. 1, January 2005, pp. 83, 84.

${ }^{25}$ Kozaki, S., "Scattering of a Gaussian beam by a homogeneous dielectric cylinder", J. Appl. Phys. Vol. 53, No. 11, pp. 7195- 7200 (1982)

${ }^{26}$ Adamovsky, G. and Ida, N., "Laser Beam Propagation Through Inhomogeneous Media," in Optical Technology in Fluid, Thermal, and Combustion flows III, edited by S. S. Cha, J. D. Trolinger, and M. Kawahashi, SPIE Proceedings, Vol. 3172, 1997, pp. 530-539.

${ }^{27}$ Born, M. and Wolf, E., Principles of Optics, $6{ }^{\text {th }}$ ed., Pergamon Press, New York, 1980, Chap. VIII.

${ }^{28}$ van Splunter,J. M. and vab den Berg, P. M., "Spectral theory of diffraction of electromagnetic waves by a strip in the plane interface of semi-infinite media", Can. J. Phys. Vol. 57, pp. 1148-1156 (1979). 
${ }^{29}$ Sanchez-Brea, L. M. and Salgado-Remacha, F. J.," Three-dimensional diffraction of a thin metallic cylinder illuminated in conical incidence: application to diameter estimation", Appl. Opt. Vol. 47, No. 26, pp. 4804-4811 (2008).

${ }^{30}$ Khodier, S. A., " Measurement of wire diameter by optical diffraction”, Optics \& Laser Technology Vol. 36, pp. 63-67 (2004).

${ }^{31}$ Cowan R G and Hornig D F, "The Experimental Determination of the Thickness of a Shock Front in a Gas,” J. Chem. Phys., Vol. 18, No. 8, 1950, pp. 1008-1018.

${ }^{32}$ Greene E F, Cowan R G, and Hornig D F, "The Thickness of Shock Fronts in Argon and Nitrogen and Rotational Heat Capacity Lags," J. Chem. Phys., Vol. 19, No 4, 1951, pp. 427-434.

${ }^{33}$ Siegman, A. E., Lasers, University Science Books, Mill Valley, CA, 1986, Chap. 17.

${ }^{34}$ WinXTest/32 Diagnostics Software, Princeton Instruments, User Manual 4411-0103 Version 2.5.E, July 25,2005 , p. 39. 